\title{
Speeds of Propagation in Classical and Relativistic Extended Thermodynamics
}

\author{
Ingo Müller \\ Technical University Berlin \\ Thermodynamik \\ 10623 Berlin \\ email: ingo.mueller@alumni.tu-berlin.de \\ http://www . thermodynamik.tu-berlin.de \\ Living Reviews in Relativity \\ ISSN 1433-8351
}

Accepted on 1 March 1999

Published on 14 June 1999

\begin{abstract}
The Navier-Stokes-Fourier theory of viscous, heat-conducting fluids provides parabolic equations and thus predicts infinite pulse speeds. Naturally this feature has disqualified the theory for relativistic thermodynamics which must insist on finite speeds and, moreover, on speeds smaller than $c$. The attempts at a remedy have proved heuristically important for a new systematic type of thermodynamics: Extended thermodynamics. That new theory has symmetric hyperbolic field equations and thus it provides finite pulse speeds.

Extended thermodynamics is a whole hierarchy of theories with an increasing number of fields when gradients and rates of thermodynamic processes become steeper and faster. The first stage in this hierarchy is the 14-field theory which may already be a useful tool for the relativist in many applications. The 14 fields - and further fields - are conveniently chosen from the moments of the kinetic theory of gases.

The hierarchy is complete only when the number of fields tends to infinity. In that case the pulse speed of non-relativistic extended thermodynamics tends to infinity while the pulse speed of relativistic extended thermodynamics tends to $c$, the speed of light.

In extended thermodynamics symmetric hyperbolicity - and finite speeds - are implied by the concavity of the entropy density. This is still true in relativistic thermodynamics for a privileged entropy density which is the entropy density of the rest frame for non-degenerate gases.

NB: This review will not be updated anymore. In May 2009, the article was republished in the revised Living Reviews layout, therefore the pagination has changed. The publication number lrr-1999-1 has not been altered.
\end{abstract}




\section{Imprint / Terms of Use}

Living Reviews in Relativity is a peer reviewed open access journal published by the Max Planck Institute for Gravitational Physics, Am Mühlenberg 1, 14476 Potsdam, Germany. ISSN 1433-8351.

Because a Living Reviews article can evolve over time, we recommend to cite the article as follows:

Ingo Müller,

"Speeds of Propagation in Classical and Relativistic Extended Thermodynamics",

Living Rev. Relativity, 2, (1999), 1. [Online Article]: cited [<date $>$ ],

http://www.livingreviews.org/lrr-1999-1

The date given as $<$ date $>$ then uniquely identifies the version of the article you are referring to.

\section{Article Revisions}

Living Reviews supports two different ways to keep its articles up-to-date:

Fast-track revision A fast-track revision provides the author with the opportunity to add short notices of current research results, trends and developments, or important publications to the article. A fast-track revision is refereed by the responsible subject editor. If an article has undergone a fast-track revision, a summary of changes will be listed here.

Major update A major update will include substantial changes and additions and is subject to full external refereeing. It is published with a new publication number.

For detailed documentation of an article's evolution, please refer always to the history document of the article's online version at http://www.livingreviews .org/lrr-1999-1. 


\section{Contents}

1 Introduction $\quad 5$

2 Scope and Structure, Characteristic Speeds $\quad 7$

2.1 Thermodynamic processes . . . . . . . . . . . . . . . . . . . 7

2.2 Elements of the constitutive theory . . . . . . . . . . . . . . . . . 8

2.3 Exploitation of the entropy inequality, Lagrange multipliers . . . . . . . . . . . . . 9

2.4 Characteristic speeds . . . . . . . . . . . . . . . . . . . . 10

3 Finite Speeds in Non-Relativistic Extended Thermodynamics 11

3.1 Concavity of the entropy density . . . . . . . . . . . . . . . . . . 11

3.2 Symmetric hyperbolicity . . . . . . . . . . . . . . . . . . . 12

3.3 Moments as variables . . . . . . . . . . . . . . . . . . . . . . . . . . . . . . . . . . . . . . . .

3.4 Specific form of the phase density . . . . . . . . . . . . . . . . . . . 13

3.5 Pulse speeds in a non-degenerate gas in equilibrium . . . . . . . . . . . . . . . . . 15

3.6 A lower bound for the pulse speed of a non-degenerate gas . . . . . . . . . . . 15

4 Finite Speeds in Relativistic Extended Thermodynamics $\quad 18$

4.1 Concavity of a privileged entropy density . . . . . . . . . . . . . . . . . . . . . . . . . . . . . .

4.2 Symmetric hyperbolicity . . . . . . . . . . . . . . . . . . . . . . 19

4.3 Moments as four-fluxes and the vector potential . . . . . . . . . . . . . . . . 20

4.4 Upper and lower bounds for the pulse speed . . . . . . . . . . . . . . . . . . . 21

5 Relativistic Thermodynamics of Gases. 14-Field Theory 23

5.1 Thermodynamic processes in viscous, heat-conducting gases . . . . . . . . . . . . 23

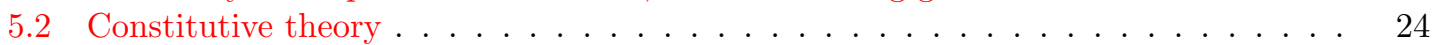

5.3 Results of the constitutive theory . . . . . . . . . . . . . . . . . . . . . . . . . . . . . . . . . . .

5.4 The laws of Navier-Stokes and Fourier . . . . . . . . . . . . . . . . . . . . . . 26

5.5 Specific results for a non-degenerate relativistic gas . . . . . . . . . . . . . . . . . . 27

5.6 Characteristic speeds in a viscous, heat-conducting gas . . . . . . . . . . . . . . 29

5.7 Discussion . . . . . . . . . . . . . . . . . . . . 29

$\begin{array}{lr}\text { References } & \mathbf{3 0}\end{array}$

\section{List of Tables}

1 Pulse speed in extended thermodynamics of moments. $n$ : Number of moments, $N$ : Highest degree of moments, $V_{\max } / c_{0}$ : Pulse speed. $\ldots \ldots \ldots$ 



\section{Introduction}

Relativistic thermodynamics is needed, because in relativity the mass of a body depends on how hot it is and the temperature is not necessarily homogeneous in equilibrium. But unlike classical thermodynamics the relativistic theory cannot be constructed on the intuitive notions of heat and work, because our intuition does not work well with relativistic effects. Therefore we must rely upon logic, or what would seem logical: the cautious and careful extrapolation of the tenets of non-relativistic thermodynamics.

A pioneer of this strategy was Carl Eckart [14, 15, 16] who - as early as 1940 - established the thermodynamics of irreversible processes, a theory now universally known by the acronym TIP. The third of Eckart's three papers addresses the relativistic theory of a fluid. Eckart's theory is an important step away from equilibria toward non-equilibrium processes. It provides the NavierStokes equations for the deviatoric stress and a generalization of Fourier's law of heat conduction. The latter permits a heat flux to be generated by an acceleration, or a temperature gradient to be equilibrated by a gravitational field.

But Eckart's theories - the relativistic and non-relativistic ones - have one draw-back: They lead to parabolic equations for the temperature and velocity and thus predict infinite pulse speeds. Naturally relativists, who know that no speed can exceed $c$, are particularly disturbed by this result and they like to call it a paradox.

Cattaneo [7] proposed a solution of the paradox as far as it concerns heat conduction ${ }^{1}$. He reasoned that under rapid changes of temperature the heat flux is somewhat influenced by the history of the temperature gradient and he was thus able to produce a hyperbolic equation for the temperature - actually a telegraph equation. Müller [35, 37] incorporated this idea into TIP and came up with a fully hyperbolic system for temperature and velocity. He calculated the pulse speeds and found them to be of the order of magnitude of the speed of sound, far removed from c. And indeed, neither Cattaneo's nor Müller's arguments have anything to do with relativity, although Müller [35] also formulated his theory relativistically. The theory became known as Extended Thermodynamics, because the canonical list of fields - density, velocity, temperature is extended in this theory to include stress and heat flux, 14 fields altogether.

The pulse speed problem may not be the most important question in thermodynamics but it is a question that can be answered, and has to be answered, and so there was a series of papers on the problem all using extended thermodynamics of 14 fields. Israel [21] - who reinvented extended thermodynamics in 1976 - and Kranys [24] and Stewart [46], and Boillat [2], and Seccia \& Strumia [44] all calculate the pulse speed for classical as well as for relativistic gases, degenerate and non-degenerate, for Bosons and Fermions, and for the ultra-relativistic case. Actually in some of these gases the pulse speed reaches the order of magnitude of $c$ but it never exceeds it.

So far, so good! But now consider this: The 14 fields mentioned above are the first moments in the kinetic theory of gases and the kinetic theory knows many more moments. In fact, in the kinetic theory we may define infinitely many moments of an increasing tensorial rank. And so Müller and his co-workers, particularly Kremer [25, 26], Weiss [49, 51, 50] and Struchtrup [47], came to realize that the original extended thermodynamics was not extended far enough. Guided by the kinetic theory of gases they formulated many-moment theories. These theories have proved their validity and relevance for quickly changing processes and processes with steep gradients, in particular for light scattering, sound dispersion, shock wave structure and radiation thermodynamics. And each

\footnotetext{
${ }^{1}$ It is true that Maxwell $[33,34]$ had an equation of transfer for the heat flux with a rate term just as postulated by Cattaneo 80 years later; such an equation arises naturally in the kinetic theory of gases. However, on both occasions Maxwell summarily dismisses the term as being small and uninteresting. He was interested in deriving the proportionality of heat flux and temperature gradient, Fourier's law. It is uncertain whether Maxwell was aware of the paradox. But, if he was, he did not care about it, at least not in the papers cited. It is conceivable that Maxwell, a prolific writer of letters as well as of papers, may have mentioned the paradox elsewhere. If so, the author of this review should like to learn about it.
} 
theory predicts a new pulse speed. Weiss [49], working with the non-relativistic kinetic theory of gases, demonstrated that the pulse speed increases with an increasing number of moments.

Boillat \& Ruggeri [4] proved this observation and - very recently - Boillat \& Ruggeri [5] also proved that the pulse speed tends to infinity in the non-relativistic kinetic theory as the number of moments becomes infinite. As yet unpublished is the corresponding result by Boillat \& Ruggeri $[6,3]$ in the relativistic case by which the pulse speed tends to $c$ as the number of moments increases. These results put an end to the long-standing paradox of pulse speeds - 50 years after Cattaneo; they are reviewed in Section 3 and 4.

The quest for macroscopic field theories with finite pulse speeds has proved heuristically useful for the discovery of the formal structure of thermodynamics, relativistic and otherwise. This structure implies

- basis equations are of balance type; hence there is the possibility of weak solutions and shocks,

- constitutive equations are local in space-time; hence follow quasilinear first-order field equations,

- entropy inequality with a concave entropy density; this implies symmetric hyperbolic field equations.

The latter property is essential for finite speeds and for the well-posedness of initial value problems which is a feature at least as desirable as finite speeds. The formal structure of the theory is described in Section 2; it was constructed by Ruggeri and his co-workers, particularly Strumia and Boillat, see $[43,41,4]$. A convenient presentation may be found in the book by Müller \& Ruggeri [39] of which a second edition has just appeared [40].

Section 5 presents extended thermodynamics of viscous, heat-conducting gases due to Liu, Müller \& Ruggeri [31], a theory of 14 fields. That section demonstrates the restrictive character of the thermodynamic constitutive theory by showing that most constitutive coefficients can be reduced to the thermal equation of state. Also new insight is provided into the form of the transport coefficients: bulk- and shear-viscosity, and thermal conductivity, which are all explicitly related here to the relaxation times of the gas.

This whole review is concerned with a macroscopic theory: Extended thermodynamics. It is true that some of the tenets of extended thermodynamics are strongly motivated by the kinetic theory of gases, for instance the choice of moments as variables. But even so, extended thermodynamics is a field theory in its own right, it is not kinetic theory.

The kinetic theory, complete with Boltzmann equation and Stoßzahlansatz, offers another possibility of discussing finite propagation speeds - or speeds smaller than $c$ in the relativistic case. Such discussions are more directly based on the observation that the atoms cannot be faster than c. Thus Cercignani [8] has directly linked the phase speed of small harmonic waves to the speed of particles and proved that the phase speeds are smaller than $c$. Cercignani \& Majorana in a follow-up paper [9] have exploited the full dispersion relation to calculate phase speeds and attenuation as functions of frequency, albeit for a simplified collision term. Earlier works on the kinetic theory which address the question of propagation speeds include Sirovich \& Thurber [45] and Wang Chang \& Uhlenbeck [48]. These works, however, are not subjects of this review. 


\section{Scope and Structure, Characteristic Speeds}

This section explains the formal structure of modern extended thermodynamics, relativistic or otherwise. Its key ingredients are

- field equations of balance type

- local constitutive equations

- entropy balance inequality

- concavity of entropy density

Thermodynamic processes are defined and characteristic speeds and the pulse speed are introduced.

\subsection{Thermodynamic processes}

Thermodynamics, and in particular relativistic thermodynamics is a field theory with the primary objective to determine the thermodynamic fields. These are typically the 14 fields of the number density of particles, the particle flux vector and the fields of the stress-energy-momentum tensor. However, in extended thermodynamics we have generally more fields and therefore it is better - at least for the initial arguments - to leave the number of fields and their tensorial character unspecified. Therefore we consider $n$ fields, combined in the $n$-vector $\boldsymbol{u}\left(x^{D}\right) . x^{D}$ denotes the space-time components of an event. We have $x^{0}=c t$ and $x^{d}=\left(x^{1}, x^{2}, x^{3}\right)^{2}$.

For the determination of the $n$ fields $\boldsymbol{u}$ we need field equations - generally $n$ of them - and these are based on the equations of balance of mechanics and thermodynamics. The generic form of these balance equations reads

$$
\boldsymbol{F}_{, A}^{A}=\boldsymbol{\pi} .
$$

The comma denotes partial differentiation with respect to $x^{A}$, and $\boldsymbol{F}^{0}$ is the $n$-vector of densities, while $\boldsymbol{F}^{a}$ is the $n$-vector of flux components. Thus $\boldsymbol{F}^{A}$ represents $n$ four-fluxes, and $\boldsymbol{\pi}$ is the $n$-vector of productions.

Obviously the balance equations (1) are not field equations for the fields $\boldsymbol{u}$, at least not in this form. They must be supplemented by constitutive equations. These relate the four-fluxes $\boldsymbol{F}^{A}$ and the productions $\boldsymbol{\pi}$ to the fields $\boldsymbol{u}$ in a materially dependent manner. We write

$$
\boldsymbol{F}^{A}=\widehat{\boldsymbol{F}}^{A}(\boldsymbol{u}) \text { and } \boldsymbol{\pi}=\widehat{\boldsymbol{\pi}}(\boldsymbol{u}) .
$$

$\widehat{\boldsymbol{F}}^{A}$ and $\widehat{\boldsymbol{\pi}}$ denote the constitutive functions. Note that the constitutive quantities $\boldsymbol{F}^{A}$ and $\boldsymbol{\pi}$ at one event depend only on the values of $\boldsymbol{u}$ at that same event. In particular there is no dependence on gradients and time derivatives of $\boldsymbol{u}$.

If the constitutive functions $\widehat{\boldsymbol{F}}^{A}$ and $\widehat{\boldsymbol{\pi}}$ are explicitly known, we may eliminate $\boldsymbol{F}^{A}$ and $\boldsymbol{\pi}$ between the balance equations (1) and the constitutive relations (2) and obtain a set of explicit field equations for the fields $\boldsymbol{u}$. These are quasilinear partial differential equations of first order. Every solution of the field equations is called a thermodynamic process.

\footnotetext{
${ }^{2}$ Throughout this work we stick to Lorentz frames, so that the metric tensor $\boldsymbol{g}$ has only diagonal components with $g_{00}=1, g_{11}=g_{22}=g_{33}=-1$.
} 


\subsection{Elements of the constitutive theory}

Since, however, the constitutive functions $\widehat{\boldsymbol{F}}^{A}$ and $\widehat{\boldsymbol{\pi}}$ are generally not explicitly known, the major task of thermodynamics is the determination of these functions, or at least the restriction of their generality. In simple cases it is possible to reduce the constitutive functions to a few coefficients which may be turned over to the experimentalist for measurement. The formulation and exploitation of such restrictions is the subject of the constitutive theory.

The tools of the constitutive theory are certain universal physical principles which have come to be accepted by the extrapolation of common experience. Above all there are three such principles:

The Entropy Inequality. The entropy density $h^{0}$ and the entropy flux $h^{a}$ combine to form a four-vector $h^{A}=\left(c h^{0}, h^{a}\right)$, whose divergence $h_{, A}^{A}$ is equal to the entropy production $\Sigma$. The four-vector $h^{A}$ and $\Sigma$ are both constitutive quantities and $\Sigma$ is assumed non-negative for all thermodynamic processes. Thus we may write $h^{A}=\hat{h}^{A}(u), \Sigma=\hat{\Sigma}(u)$ and

$$
h^{A}{ }_{, A}=\Sigma \geq 0 \quad \forall \quad \text { thermodynamic processes. }
$$

This inequality is clearly an extrapolation of the entropy inequalities known in thermostatics and thermodynamics of irreversible processes; it was first stated in this generality by Müller $[36,38]$.

The Principle of Relativity. The principle of relativity requires that the field equations and the entropy inequality have the same form in all

- Galilei frames for the non-relativistic case, or in all

- Lorentz frames for the relativistic case.

The formal statement and exploitation of this principle have to await a specific choice for the fields $\boldsymbol{u}$ and the four-fluxes $\boldsymbol{F}^{A}$.

The Requirement of Concavity of the Entropy Density. It is possible, and indeed common, to make a specific choice for the fields $\boldsymbol{u}$ and the concavity postulate is contingent upon that choice.

- In the non-relativistic case we choose the fields $\boldsymbol{u}$ as the densities $\boldsymbol{F}^{0}$. The requirement of concavity demands that the entropy density $h^{0}$ be a concave function of the variables $\boldsymbol{F}^{0}$ :

$$
\frac{\partial^{2} h^{0}}{\partial \boldsymbol{F}^{0} \partial \boldsymbol{F}^{0}} \sim \text { negative definite. }
$$

- In the relativistic case we choose the fields $\boldsymbol{u}$ as the densities $\boldsymbol{F}_{\zeta}=\boldsymbol{F}^{A} \zeta_{A}$ in a generic Lorentz frame that moves with the four-velocity $c \zeta_{A}$ with respect to the observer. We have $\zeta^{A} \zeta_{A}=1$ and $\zeta^{0}>0$. We cannot be certain that in all these frames the entropy density $h_{\zeta}=h^{A} \zeta_{A}$ is concave as a function of $\boldsymbol{F}_{\zeta}$. Therefore we assume that there is at least one $\zeta_{A}$ - a privileged one, denoted by $\bar{\zeta}_{A}-$ such that $h_{\bar{\zeta}}=h^{A} \bar{\zeta}_{A}$ is concave with respect to $\boldsymbol{F}_{\bar{\zeta}}=\boldsymbol{F}^{A} \zeta_{A}$, viz.

$$
\frac{\partial^{2} h_{\bar{\zeta}}}{\partial \boldsymbol{F}_{\bar{\zeta}} \partial \boldsymbol{F}_{\bar{\zeta}}} \sim \text { negative definite. }
$$

The privileged co-vector $\bar{\zeta}_{A}$ remains to be chosen, see Section 4.1. 
In both cases the concavity postulate makes it possible that the entropy be maximal for a particular set of fields - the set corresponding to equilibrium - and that is its attraction for physicists. For mathematicians the attraction of the concavity postulate lies in the observation that concavity implies symmetric hyperbolicity of the field equations, see Sections 3.2 and 4.2 below.

\subsection{Exploitation of the entropy inequality, Lagrange multipliers}

The key to the exploitation of the entropy inequality lies in the fact that the inequality should hold for thermodynamic processes, i.e. solutions of the field equations rather than for all fields. By a theorem proved by Liu [30] this constraint may be removed by the use of Lagrange multipliers $\boldsymbol{\Lambda}$ - themselves constitutive quantities, so that $\boldsymbol{\Lambda}=\widehat{\boldsymbol{\Lambda}}(\boldsymbol{u})$ holds. Indeed, the new inequality

$$
h^{A}{ }_{, A}-\boldsymbol{\Lambda} \cdot\left(\boldsymbol{F}^{A}{ }_{, A}-\boldsymbol{\pi}\right) \geq 0 \quad \forall \text { fields } \boldsymbol{u} .
$$

is equivalent to (3).

Liu's proof proceeds from the observation that the field equations and the entropy equation are linear functions of the derivatives $\boldsymbol{u}_{, A}$. By the Cauchy-Kowalewski theorem these derivatives are local representatives of an analytical thermodynamic process and therefore the entropy principle requires that the field equations and the entropy equation must hold for all $\boldsymbol{u}_{, A}$. It is then a simple problem of linear algebra to prove that

$$
\frac{\partial h^{A}}{\partial \boldsymbol{u}} \text { must be a linear combination of } \frac{\partial \boldsymbol{F}^{A}}{\partial \boldsymbol{u}} .
$$

Liu's proof is not restricted to quasilinear systems of first order equations but here we need his result only in that particularly simple case.

We may use the chain rule on $h^{A}=\hat{h}^{A}(\boldsymbol{u})$ and $\boldsymbol{F}^{A}=\widehat{\boldsymbol{F}}^{A}(\boldsymbol{u})$ in (6) and obtain

$$
\left(\frac{\partial h^{A}}{\partial \boldsymbol{u}}-\boldsymbol{\Lambda} \cdot \frac{\partial \boldsymbol{F}^{A}}{\partial \boldsymbol{u}}\right) \boldsymbol{u}_{, A}+\boldsymbol{\Lambda} \cdot \boldsymbol{\pi} \geq 0 .
$$

The left hand side is an explicit linear function of the derivatives $\boldsymbol{u}_{, A}$ and, since the inequality must hold for all fields $\boldsymbol{u}$, it must hold in particular for arbitrary values of the derivatives $\boldsymbol{u}_{A_{A}}$. The entropy inequality could thus easily be violated by some choice of $\boldsymbol{u}_{, A}$ unless we have

$$
d h^{A}=\boldsymbol{\Lambda} \cdot d \boldsymbol{F}^{A}
$$

and there remains the residual inequality

$$
\Lambda \cdot \pi \geq 0
$$

The differential forms (8) represent a generalization of the Gibbs equation of equilibrium thermodynamics; the classical Gibbs equation for the entropy density is here generalized into four equations for the entropy four-flux. Relation (9) is the residual entropy inequality which represents the irreversible entropy production. Note that the entropy production is entirely due to the production terms in the balance equations. 


\subsection{Characteristic speeds}

The system of field equations (1), (2) may be written as a quasilinear system of $n$ equations in the form

$$
\frac{\partial \boldsymbol{F}^{A}}{\partial \boldsymbol{u}} \boldsymbol{u}, A=\boldsymbol{\pi}
$$

Such a system allows the propagation of weak waves, so-called acceleration waves. There are $n$ such waves and their speeds are called characteristic speeds, which are not necessarily all different. The fastest characteristic speed is the pulse speed. This is the largest speed by which information can propagate.

Let $\phi\left(x^{D}\right)=0$ define the wave front; thus

$$
\frac{\partial \phi}{\partial x^{a}}=|\operatorname{grad} \phi| n_{a} \quad \text { and } \quad \frac{\partial \phi}{\partial c t}=-|\operatorname{grad} \phi| \frac{V}{c}
$$

define its unit normal $\boldsymbol{n}$ and the speed $V$. An easy manipulation provides

$$
\frac{V^{2}}{c^{2}}=1+\frac{g^{A B} \phi, A \phi, B}{|\operatorname{grad} \phi|^{2}}
$$

Since in a weak wave the fields $\boldsymbol{u}$ have no jump across the front, the jumps in the gradients must have the direction of $\boldsymbol{n}$ and we may write

$$
\left[\boldsymbol{u}_{, a}\right]=\delta \boldsymbol{u} n_{a}, \quad\left[\boldsymbol{u}_{, 0}\right]=-\frac{V}{c} \delta \boldsymbol{u}, \text { where } \delta \boldsymbol{u}=\left[n_{a} \frac{\partial \boldsymbol{u}}{\partial x^{a}}\right] .
$$

$\delta \boldsymbol{u}$ is the magnitude of the jump of the gradient of $\boldsymbol{u}$. The square brackets denote differences between the front side and the back side of the wave.

In the field equations (10) the matrix $\frac{\partial \boldsymbol{F}^{A}}{\partial \boldsymbol{u}}$ and the productions are equal on both sides of the wave, since both only depend on $\boldsymbol{u}$ and since $\boldsymbol{u}$ is continuous. Thus, if we take the difference of the equations on the two sides and use (13) and (11), we obtain

$$
\phi, A \frac{\partial \boldsymbol{F}^{A}}{\partial \boldsymbol{u}} \delta \boldsymbol{u}=0
$$

Non-trivial solutions for $\delta \boldsymbol{u}$ require that this linear homogeneous system have a vanishing determinant

$$
\operatorname{det}\left(\phi, A \frac{\partial \boldsymbol{F}^{A}}{\partial \boldsymbol{u}}\right)=0
$$

Insertion of (11) into (15) provides an algebraic equation for $V$ whose solutions - for a prescribed direction $\boldsymbol{n}$-determine $n$ wave speeds $V$, of which the largest one is the pulse speed. Equation (15) is called the characteristic equation of the system (10) of field equations. By (11) it may be written in the form

$$
\operatorname{det}\left(\frac{\partial \boldsymbol{F}^{a}}{\partial \boldsymbol{u}} n_{a}-\frac{V}{c} \frac{\partial \boldsymbol{F}^{0}}{\partial \boldsymbol{u}}\right)=0 .
$$




\section{Finite Speeds in Non-Relativistic Extended Thermody- namics}

It is shown in this section that the concavity of the entropy density $h^{0}$ with respect to the fields $\boldsymbol{F}^{0}$ implies global invertibility of the map $\boldsymbol{F}^{0} \Longleftrightarrow \boldsymbol{\Lambda}$, where $\boldsymbol{\Lambda}$ is the $n$-vector of Lagrange multipliers. Also the system of field equations - written in terms of $\boldsymbol{\Lambda}$ - is recognized as a symmetric hyperbolic system which guarantees

- finite characteristic speeds and

- well-posedness of initial value problems.

Thus we conclude that no paradox of infinite speeds can arise in extended thermodynamics, at least not for finitely many variables.

A commonly treated special case occurs when the fields $\boldsymbol{u}$ are moments of the phase density of a gas. In this case the pulse speed depends on the degree of extension, i.e. on the number $n$ of fields $\boldsymbol{u}$. For a gas in equilibrium the pulse speeds can be calculated for any $n$. Also it can be estimated that the pulse speed tends to infinity as $n$ grows to infinity.

\subsection{Concavity of the entropy density}

We recall the argument of Section 2.2 concerning concavity and choose the fields $\boldsymbol{u}$ to mean the fields of densities $\boldsymbol{F}^{0}$. Thus equation (8), for $A=0$, leads to

$$
\boldsymbol{\Lambda}=\frac{\partial h^{0}}{\partial \boldsymbol{F}^{0}}, \text { hence } \frac{\partial \boldsymbol{\Lambda}}{\partial \boldsymbol{F}^{0}}=\frac{\partial^{2} h^{0}}{\partial \boldsymbol{F}^{0} \partial \boldsymbol{F}^{0}} .
$$

Therefore the concavity of the entropy density $h^{0}$ in the variables $\boldsymbol{F}^{0}-$ the negative-definiteness of $\frac{\partial^{2} h^{0}}{\partial \boldsymbol{F}^{0} \partial \boldsymbol{F}^{0}}-$ implies global invertibility between the field vector $\boldsymbol{F}^{0}$ and the Lagrange multipliers $\Lambda$.

The transformation $\boldsymbol{F}^{0} \Longleftrightarrow \boldsymbol{\Lambda}$ helps us to recognize the structure of the field equations and to find generic restrictions on the constitutive functions.

Indeed, obviously, with $\boldsymbol{\Lambda}$ as field vector instead of $\boldsymbol{u}$, or $\boldsymbol{F}^{0}$, we may rephrase (8) in the form

$$
d h^{\prime A}=\boldsymbol{F}^{A} d \boldsymbol{\Lambda},
$$

where

$$
h^{\prime A} \equiv \boldsymbol{\Lambda} \cdot \boldsymbol{F}^{A}-h^{A} .
$$

Thus we have

$$
\boldsymbol{F}^{A}=\frac{\partial h^{\prime A}}{\partial \boldsymbol{\Lambda}},
$$

and

$$
h^{A}=\mathbf{\Lambda} \frac{\partial h^{\prime A}}{\partial \boldsymbol{\Lambda}}-h^{\prime A},
$$

so that the constitutive quantities $\boldsymbol{F}^{A}$ and $h^{A}$ result from $h^{\prime A}$ - defined by equation (19) - through differentiation. Therefore the vector $h^{\prime A}$ is called the thermodynamic vector potential.

It follows from equation (20) that

$$
\frac{\partial \boldsymbol{F}^{A}}{\partial \boldsymbol{\Lambda}} \text { is symmetric, }
$$

which implies $4 n(n-1)$ restrictions on the constitutive functions $\widehat{\boldsymbol{F}}^{A}(\boldsymbol{\Lambda})$. 


\subsection{Symmetric hyperbolicity}

Using the new variables $\boldsymbol{\Lambda}$ we may write the field equations in the form

$$
\frac{\partial \boldsymbol{F}^{A}}{\partial \boldsymbol{\Lambda}} \boldsymbol{\Lambda}_{, A}=\boldsymbol{\pi}(\boldsymbol{\Lambda})
$$

or, by (20):

$$
\frac{\partial^{2} \boldsymbol{h}^{\prime A}}{\partial \boldsymbol{\Lambda} \partial \boldsymbol{\Lambda}} \boldsymbol{\Lambda}_{, A}=\boldsymbol{\pi}(\boldsymbol{\Lambda}) .
$$

We observe that the coefficient matrices in (23) are Hessian matrices derived from the vector potential $h^{\prime A}$. Therefore the matrices are symmetric.

Also the matrix $\frac{\partial^{2} h^{\prime 0}}{\partial \boldsymbol{\Lambda} \partial \boldsymbol{\Lambda}}$ is negative definite on account on the concavity (4) of $h^{0}$ with respect to $\boldsymbol{F}^{0}$. This is so, because the defining equation of $h^{\prime 0}$, viz.

$$
h^{\prime 0}=\boldsymbol{\Lambda} \cdot \boldsymbol{F}^{0}-h^{0}
$$

represents the Legendre transformation from $h^{0}$ to $h^{\prime 0}$ connected with the map $\boldsymbol{F}^{0} \Longleftrightarrow \boldsymbol{\Lambda}$ between dual fields. Indeed, we have by $(20,21)$ and (8)

$$
\boldsymbol{F}^{0}=\frac{\partial h^{\prime 0}}{\partial \boldsymbol{\Lambda}} \quad \text { and } \quad \boldsymbol{\Lambda}=\frac{\partial h^{0}}{\partial \boldsymbol{F}^{0}}
$$

Such a transformation preserves convexity - or concavity - so that $h^{\prime 0}$ is a concave function of $\boldsymbol{\Lambda}$, since $h^{0}$ is a concave function of $\boldsymbol{F}^{0}$.

A quasilinear system of the type (23) with symmetric coefficient matrices, of which the temporal one is definite, is called symmetric hyperbolic. We conclude that symmetric hyperbolicity of the equations (23) for the fields $\boldsymbol{\Lambda}$ is equivalent to the concavity of the entropy density $h^{0}$ in terms of the fields of densities $\boldsymbol{F}^{0}$.

Hyperbolicity implies finite characteristic speeds, and symmetric hyperbolic systems guarantee the well-posedness of initial value problems, i.e. existence and uniqueness of solutions - at least in the neighbourhood of an event - and continuous dependence on the data.

Thus without having actually calculated a single characteristic speed, we have resolved Cattaneo's paradox of infinite speeds. The structure of extended thermodynamics guarantees that all speeds are finite; no paradox can occur!

The fact that a system of balance-type field equations is symmetric hyperbolic, if it is compatible with the entropy inequality and the concavity of the entropy density was discovered by Godunov [19] in the special case of Eulerian fluids. In general this was proved by Boillat [1]. Ruggeri \& Strumia [43] have found that the symmetry is revealed only when the Lagrange multipliers are chosen as variables; these authors were strongly motivated by Liu's results of 1972 and by a paper by Friedrichs \& Lax [18] which appeared a year earlier.

\subsection{Moments as variables}

In a gas the most plausible choice for the four-fluxes $\boldsymbol{F}^{A}$ are the moments of the phase density $f(\boldsymbol{x}, \boldsymbol{p}, t)$ of the atoms. Thus we have

$$
F_{\alpha}^{A}=\int p^{A} p_{\alpha} f d \boldsymbol{p}, \quad(\alpha=1,2, \ldots n),(A=0,1,2,3) .
$$


$p^{0}$ is equal to $m c$, where $m$ is the atomic mass, while $p^{a}$ denotes the Cartesian coordinates of the momentum of an atom. $\alpha$ is a multi-index and $p_{\alpha}$ stands for

$$
p_{\alpha}= \begin{cases}1 & \alpha=1 \\ p^{i_{1}} & \alpha=2,3,4 \\ p^{i_{1}} p^{i_{2}} & \alpha=5,6, \ldots 10 \\ p^{i_{1}} p^{i_{2}} \ldots p^{i_{N}} & \alpha=n-\frac{1}{2}(N+1)(N+2), \ldots, n\end{cases}
$$

so that the densities $F_{\alpha}^{0},(\alpha=1,2, \ldots n)$ form a hierarchy of moments of increasing tensorial degree up to degree $N$. Because of the evident symmetry of (27) there is a relation between $n$ and $N$, viz.

$$
n=\frac{1}{6}(N+1)(N+2)(N+3) .
$$

The kinetic theory of gases implies that the moments (26) satisfy equations of balance of the type (1) so that the foregoing analysis holds. In particular, we have (18) which may now be written in the form

$$
\begin{gathered}
d h^{\prime A}=F_{\alpha}^{A} d \Lambda_{\alpha}= \\
\int p^{A} d\left(\Lambda_{\alpha} p_{\alpha}\right) f d \boldsymbol{p}= \\
\int p^{A} d F\left(\Lambda_{\alpha} p_{\alpha}\right) d \boldsymbol{p}= \\
d \int p^{A} F\left(\Lambda_{\alpha} p_{\alpha}\right) d \boldsymbol{p} .
\end{gathered}
$$

We introduce $\chi=\Lambda_{\alpha} p_{\alpha}$ and note that by (30) the phase density depends on the single variable $\chi$ only. Also (32) implies that the vector potential has the form

$$
h^{\prime A}=\int p^{A} F(\chi) d \boldsymbol{p}
$$

where, by (31), $\frac{d F}{d \chi}=f$ holds. The field equations (23) now read

$$
\left[\int p^{A} p_{\alpha} p_{\beta} \frac{d^{2} F}{d \chi^{2}} d p\right] \Lambda_{\beta, A}=\pi_{\alpha}
$$

Obviously the coefficient matrices are symmetric in $\alpha, \beta$ and $\int p_{\alpha} p_{\beta} \frac{d^{2} F}{d \chi^{2}} d \boldsymbol{p}$ is negative definite, provided that

$$
\frac{d^{2} F}{d \chi^{2}}<0
$$

i.e. $F(\chi)$ must be concave for the system (34) to be symmetric hyperbolic.

\subsection{Specific form of the phase density}

For moments as variables the entropy four-flux $h^{A}$ follows from (19) and (33). We obtain

$$
h^{A}=\int p^{A}(\chi f(\chi)-F(\chi)) d \boldsymbol{p} .
$$


On the other hand statistical mechanics defines the four-flux of entropy by (e.g. see Huang [20])

$$
h^{A}=-k \int p^{A}\left(\ln \frac{f}{y} \pm \frac{y}{f}\left(1 \pm \frac{f}{y}\right) \ln \left(1 \pm \frac{f}{y}\right)\right) f d \boldsymbol{p} \text { for } \begin{gathered}
\text { Fermions } \\
\text { Bosons }
\end{gathered}
$$

$k$ is the Boltzmann constant and $1 / y$ is the smallest phase space element.

Comparison shows that we must have

$$
\chi f(\chi)-F(\chi)=-k\left(\ln \frac{f}{y} \pm \frac{y}{f}\left(1 \pm \frac{f}{y}\right) \ln \left(1 \pm \frac{f}{y}\right)\right) f
$$

and hence, by differentiation with respect to $\chi$,

$$
f=\frac{y}{\mathrm{e}^{\chi / k} \pm 1}
$$

so that

$$
F=\mp k y \ln \left(1 \pm \mathrm{e}^{-\chi / k}\right)
$$

$f$ is the phase density appropriate to a degenerate gas in non-equilibrium. Differentiation of (39) with respect to $\chi$ proves the inequality (35).

Therefore symmetric hyperbolicity of the system (34) and hence the concavity of the entropy density with respect to the variables $F_{\alpha}^{0}$ is implied by the moment character of the fields and the form of the four-flux of entropy.

For a non-degenerate gas the term \pm 1 in the denominator of (38) may be neglected. In that case we have

$$
f=y \mathrm{e}^{-\chi / k},
$$

hence

$$
F=-k f \quad \text { and } \quad \frac{d^{2} F}{d \chi^{2}}=-\frac{1}{k} f,
$$

and therefore the field equations (23), (34) assume the form

$$
\left[-\frac{1}{k} \int p^{A} p_{\alpha} p_{\beta} f d \boldsymbol{p}\right] \Lambda_{\beta, A}=\pi_{\alpha} .
$$

Note that the matrices of coefficients are composed of moments in this case of a non-degenerate gas.

We know that a non-degenerate gas at rest in equilibrium exhibits the Maxwellian phase density

$$
f_{E}=\frac{n}{\sqrt{2 \pi m k T}^{3}} \mathrm{e}^{-\frac{p^{2}}{2 m k T}} .
$$

$n$ and $T$ denote the number density and the temperature of the gas in equilibrium. Comparison of (43) with (40) shows that only two Lagrange multipliers are non-zero in equilibrium, viz.

$$
\Lambda^{E}=k \ln \frac{y \sqrt{2 \pi m k T}^{3}}{n} \text { and } \quad \Lambda_{i i}^{E}=\frac{1}{\frac{2}{3} m k T} .
$$




\subsection{Pulse speeds in a non-degenerate gas in equilibrium}

We recall the discussion of characteristic speeds in Section 2.4 which we apply to the system (23) of field equations. The characteristic equation of this system reads

$$
\operatorname{det}\left(\phi, A \frac{\partial^{2} h^{\prime A}}{\partial \boldsymbol{\Lambda} \partial \boldsymbol{\Lambda}}\right)=0
$$

or, by (11):

$$
\operatorname{det}\left(\frac{\partial^{2} h^{\prime a}}{\partial \boldsymbol{\Lambda} \partial \boldsymbol{\Lambda}} n_{a}-\frac{V}{c} \frac{\partial^{2} h^{\prime 0}}{\partial \boldsymbol{\Lambda} \partial \boldsymbol{\Lambda}}\right)=0 .
$$

This equation determines the characteristic speeds $V$, whose maximal value $V_{\max }$ is the pulse speed. In the case of moments and for a non-degenerate gas at rest and in equilibrium this equation reads, by $(42)$,

$$
\operatorname{det}\left(\int\left(p^{a} n_{a}-V m\right) p_{\alpha} p_{\beta} f_{E} d \boldsymbol{p}\right)=0 .
$$

$f_{E}$ is the Maxwellian phase density, so that all integrals in (47) are Gaussian integrals, easy to calculate. Weiss [49] has calculated the speeds $V$ for different degrees $n$ of extended thermodynamics. Recall that $\alpha, \beta$ range over the values 1 through $n$. He has made a list of $V_{\max }$ which is represented here in Table 1 . $V_{\max }$ is normalized in Table 1 by $c_{o}=\sqrt{\frac{5 k T}{3 m}}$, the ordinary speed of sound, sometimes called the adiabatic sound speed.

Inspection of Table 1 shows that the pulse speed increases monotonically with the number of moments and there is clearly a suspicion that it may tend to infinity as $n$ goes to infinity. This suspicion will presently be confirmed.

\subsection{A lower bound for the pulse speed of a non-degenerate gas}

Since in (47) the integral $\int p^{a} n_{a} p_{\alpha} p_{\beta} f_{E} d \boldsymbol{p}$ is symmetric and $\int p_{\alpha} p_{\beta} f_{E} d \boldsymbol{p}$ is symmetric and positive definite, it follows from linear algebra ${ }^{3}$ that

$$
\int\left(p^{a} n_{a}-V_{\max } m\right) p_{\alpha} p_{\beta} f_{E} d \boldsymbol{p} \quad \text { is negative semi-definite. }
$$

Boillat \& Ruggeri [5] have used this knowledge to derive an estimate for $V_{\max }$ in terms of $N$, the highest tensorial degree of the moments. The estimate reads

$$
\frac{V_{\max }}{c_{0}} \geq \sqrt{\frac{6}{5}\left(N-\frac{1}{2}\right)} .
$$

Therefore, indeed, as more and more moments are drawn into the scheme of extended thermodynamics, the pulse speed goes up and, if $N$ tends to infinity, so does $V_{\max }$.

The proof of (49) rests on the realization that - because of symmetry $-p_{\alpha}=p^{i_{1}} p^{i_{2}} \ldots p^{i_{l}}$ has only $\frac{1}{2}(l+1)(l+2)$ independent components and they are simply powers of $p^{1}, p^{2}$ and $p^{3}$, so that $p_{\alpha}$ may be written as $\left(p^{1}\right)^{p}\left(p^{2}\right)^{q}\left(p^{3}\right)^{r}$ with $p+q+r=l$. Accordingly $p_{\beta}=p^{j_{1}} p^{j_{2}} \ldots p^{j_{k}}$ may be written as $\left(p^{1}\right)^{s}\left(p^{2}\right)^{t}\left(p^{3}\right)^{u}$ with $s+t+u=k$.

Therefore (48) assumes the form

$$
\int\left(p^{a} n_{a}-V_{\max } m\right)\left(p^{1}\right)^{p+s}\left(p^{2}\right)^{q+t}\left(p^{3}\right)^{r+u} f_{E} d \boldsymbol{p} \text { - negative semi-definite. }
$$

\footnotetext{
${ }^{3}$ If the reader does not recall this theorem, he is advised to recapitulate the part of linear algebra that deals with
} the simultaneous diagonalization of two symmetric matrices. 
Table 1: Pulse speed in extended thermodynamics of moments. $n$ : Number of moments, $N$ : Highest degree of moments, $V_{\max } / c_{0}$ : Pulse speed.

\begin{tabular}{rcc||rcc}
\hline$n$ & $N$ & $V_{\max } / c_{0}$ & $n$ & $N$ & $V_{\max } / c_{0}$ \\
\hline 4 & 1 & 0.77459667 & 2600 & 23 & 6.59011627 \\
10 & 2 & 1.34164079 & 2925 & 24 & 6.75262213 \\
20 & 3 & 1.80822948 & 3276 & 25 & 6.91176615 \\
35 & 4 & 2.21299946 & 3654 & 26 & 7.06774631 \\
56 & 5 & 2.57495874 & 4060 & 27 & 7.22074198 \\
84 & 6 & 2.90507811 & 4495 & 28 & 7.37091629 \\
120 & 7 & 3.21035245 & 4960 & 29 & 7.51841807 \\
165 & 8 & 3.49555791 & 5456 & 30 & 7.66338362 \\
220 & 9 & 3.76412372 & 5984 & 31 & 7.80593804 \\
286 & 10 & 4.01860847 & 6545 & 32 & 7.94619654 \\
364 & 11 & 4.26098014 & 7140 & 33 & 8.08426549 \\
455 & 12 & 4.26098014 & 7770 & 34 & 8.22024331 \\
560 & 13 & 4.71528716 & 8436 & 35 & 8.35422129 \\
680 & 14 & 4.92949284 & 9139 & 36 & 8.48628432 \\
816 & 15 & 5.13625617 & 9880 & 37 & 8.61651144 \\
969 & 16 & 5.33629130 & 10660 & 38 & 8.74497644 \\
1140 & 17 & 5.53020569 & 11480 & 39 & 8.87174833 \\
1330 & 18 & 5.71852112 & 12341 & 40 & 8.99689171 \\
1540 & 19 & 5.90168962 & 13244 & 41 & 9.12046722 \\
1771 & 20 & 6.08010585 & 14190 & 42 & 9.24253184 \\
2024 & 21 & 6.25411673 & 15180 & 43 & 9.36313918 \\
2300 & 22 & 6.42402919 & & & \\
\hline & & & & &
\end{tabular}


The elements of a semi-definite matrix $a_{i j}$ satisfy the inequalities $a_{i i} a_{j j} \geq a_{i j}^{2}$ and therefore (50) implies

$$
\begin{aligned}
& \left(\int\left(p^{a} n_{a}-V_{\max } m\right)\left(p^{1}\right)^{2 p}\left(p^{2}\right)^{2 q}\left(p^{3}\right)^{2 r} f_{E} d \boldsymbol{p}\right) \times \\
\times & \left(\int\left(p^{a} n_{a}-V_{\max } m\right)\left(p^{1}\right)^{2 s}\left(p^{2}\right)^{2 t}\left(p^{3}\right)^{2 u} f_{E} d \boldsymbol{p}\right) \geq \\
\geq & \left(\int\left(p^{a} n_{a}-V_{\max } m\right)\left(p^{1}\right)^{2 p+s}\left(p^{2}\right)^{2 q+t}\left(p^{3}\right)^{2 r+u} f_{E} d \boldsymbol{p}\right)^{2}
\end{aligned}
$$

Since $f_{E}$ is an even function of $\boldsymbol{p}$ we obtain

$$
\begin{aligned}
& \left(V_{\max }\right)^{2} m^{2}\left(\int\left(p^{1}\right)^{2 p}\left(p^{2}\right)^{2 q}\left(p^{3}\right)^{2 r} f_{E} d \boldsymbol{p}\right) \times \\
\times & \left(\int\left(p^{1}\right)^{2 s}\left(p^{2}\right)^{2 t}\left(p^{3}\right)^{2 u} f_{E} d \boldsymbol{p}\right) \geq \\
\geq & \left(\int\left(p^{a} n_{a}-V_{\max } m\right)\left(p^{1}\right)^{p+s}\left(p^{2}\right)^{q+t}\left(p^{3}\right)^{r+u} f_{E} d \boldsymbol{p}\right)^{2}
\end{aligned}
$$

This estimate depends on the choice of the exponents $p$ through $u$ and we choose, rather arbitrarily $p=N, s=N-1$ and all others zero. Also we set $n_{a}=(1,0,0)$. In that case (52) implies

$$
V_{\max }^{2} \geq \frac{\int\left(p^{1}\right)^{2 N} f_{E} d p^{1}}{\left(p^{1}\right)^{2(N-1)} f_{E} d p^{1}}=\frac{6}{5} \cdot \frac{5}{3} \frac{k}{m} T\left(N-\frac{1}{2}\right)
$$

which proves (49).

An easy check will show that for each $N$ the value $\sqrt{\frac{6}{5}\left(N-\frac{1}{2}\right)}$ lies below the corresponding values of Table 1, as they must. It may well be possible to tighten the estimate (49). 


\section{Finite Speeds in Relativistic Extended Thermodynamics}

In the relativistic theory the entropy density is not a scalar, it depends on the frame. This fact creates problems: Granted that an entropy density tends to be concave, which one would that be? To my knowledge this question is unresolved. In this section we assume that there exists a privileged frame in which the entropy density is concave. And we choose the privileged frame such that symmetric hyperbolicity of the system of field equations is guaranteed. These considerations have been motivated by a paper by Ruggeri [42].

Symmetric hyperbolicity means finite characteristic speeds, not necessarily speeds smaller than the speed of light. However, for moments as four-fluxes it can be shown that all speeds are smaller or equal to $c$ and that for infinitely many moments the pulse speed tends to $c$. Moreover, for moments the privileged frame is the rest frame of the gas, at least, if the gas is non-degenerate.

\subsection{Concavity of a privileged entropy density}

We recall the arguments of Section 2.2 concerning concavity in the relativistic case and choose the fields $\boldsymbol{u}$ to mean the privileged densities $\boldsymbol{F}_{\bar{\zeta}}=\boldsymbol{F}^{A} \bar{\zeta}_{A}$. The privileged entropy density is assumed by (5) to be concave with respect to the privileged fields $\boldsymbol{F}_{\bar{\zeta}}$. The privileged co-vector $\bar{\zeta}_{A}$ will be chosen so that the concavity of $h_{\bar{\zeta}}$ implies symmetric hyperbolicity of the field equations.

From (8) we obtain after multiplication by $\bar{\zeta}_{A}$

$$
\boldsymbol{\Lambda}=\frac{\partial h_{\bar{\zeta}}}{\partial \boldsymbol{F}_{\bar{\zeta}}}+h^{\prime A} \frac{\partial \bar{\zeta}_{A}}{\partial \boldsymbol{F}_{\bar{\zeta}}},
$$

hence

$$
\frac{\partial \boldsymbol{\Lambda}}{\partial \boldsymbol{F}_{\bar{\zeta}}}=\frac{\partial^{2} h_{\bar{\zeta}}}{\partial \boldsymbol{F}_{\bar{\zeta}} \partial \boldsymbol{F}_{\bar{\zeta}}}+\frac{\partial}{\partial \boldsymbol{F}_{\bar{\zeta}}}\left(h^{\prime A} \frac{\partial \bar{\zeta}_{A}}{\partial \boldsymbol{F}_{\bar{\zeta}}}\right) .
$$

$h^{\prime A}$ is still defined as $\boldsymbol{\Lambda} \cdot \boldsymbol{F}^{A}-h^{A}$, as in (19). From (55) it follows that the concavity of $h_{\bar{\zeta}}\left(\boldsymbol{F}_{\bar{\zeta}}\right)-$ the negative definiteness of $\frac{\partial^{2} h_{\bar{\zeta}}}{\partial \boldsymbol{F}_{\bar{\zeta}} \partial \boldsymbol{F}_{\bar{\zeta}}}$-implies global invertibility between the field vector $\boldsymbol{F}_{\bar{\zeta}}$ and the Lagrange multipliers $\boldsymbol{\Lambda}$, provided that the privileged co-vector $\bar{\zeta}^{A}$ is chosen as co-linear to the vector potential $h^{\prime A}$. We set

$$
\bar{\zeta}^{A}=-\frac{h^{\prime A}}{\sqrt{h^{\prime A} h_{A}^{\prime}}}
$$

Indeed, in that case we have

$$
h^{\prime A} \frac{\partial \bar{\zeta}^{A}}{\partial \boldsymbol{F}_{\bar{\zeta}}}=0
$$

hence

$$
\bar{\zeta}^{A} \frac{\partial^{2} \bar{\zeta}^{A}}{\partial \boldsymbol{F}_{\bar{\zeta}} \partial \boldsymbol{F}_{\bar{\zeta}}}=-\frac{\partial \bar{\zeta}^{A}}{\partial \boldsymbol{F}_{\bar{\zeta}}} \frac{\partial \bar{\zeta}^{A}}{\partial \boldsymbol{F}_{\bar{\zeta}}} \sim \text { positive semi-definite }
$$

so that, by (57), the second term on the right hand side of (55) vanishes and $\frac{\partial \boldsymbol{\Lambda}}{\partial \boldsymbol{F}_{\bar{\zeta}}}$ is definite. Equation (58) will be used later.

With $\boldsymbol{\Lambda}$ as a field vector, instead of $\boldsymbol{F}_{\bar{\zeta}}$, we may rephrase (8) in the form

$$
d h^{\prime A}=\boldsymbol{F}^{A} \cdot d \boldsymbol{\Lambda},
$$

or

$$
\boldsymbol{F}^{A}=\frac{\partial h^{\prime A}}{\partial \boldsymbol{\Lambda}},
$$


hence

$$
\boldsymbol{F}_{\bar{\zeta}}=\frac{\partial h_{\bar{\zeta}}}{\partial \boldsymbol{\Lambda}}
$$

where $h_{\bar{\zeta}}^{\prime}=h^{\prime A} \bar{\zeta}^{A}=\boldsymbol{\Lambda} \cdot \boldsymbol{F}_{\bar{\zeta}}-h_{\bar{\zeta}}$. Thus $h_{\bar{\zeta}}^{\prime}$ is the Legendre transform of $h_{\bar{\zeta}}$ with respect to the map $\boldsymbol{F}_{\bar{\zeta}} \Longleftrightarrow \boldsymbol{\Lambda}$. It follows that $h_{\bar{\zeta}}^{\prime}$ is concave in $\boldsymbol{\Lambda}$, since $h_{\bar{\zeta}}$ is concave in $\boldsymbol{F}_{\bar{\zeta}}$; thus we have

$$
\frac{\partial^{2} h_{\bar{\zeta}}^{\prime}}{\partial \boldsymbol{\Lambda} \partial \boldsymbol{\Lambda}} \sim \text { negative definite. }
$$

\subsection{Symmetric hyperbolicity}

The transformation $\boldsymbol{F}_{\bar{\zeta}} \Longleftrightarrow \boldsymbol{\Lambda}$ helps us to recognize the structure of the field equations. Obviously with $\boldsymbol{\Lambda}$ as the field vector, instead of $\boldsymbol{F}_{\bar{\zeta}}$ we may rephrase the field equations (10) as

$$
\frac{\partial \boldsymbol{F}^{A}}{\partial \boldsymbol{\Lambda}} \boldsymbol{\Lambda}_{, A}=\boldsymbol{\pi}(\boldsymbol{\Lambda})
$$

or, by (60):

$$
\frac{\partial^{2} h^{\prime^{A}}}{\partial \boldsymbol{\Lambda} \partial \boldsymbol{\Lambda}} \boldsymbol{\Lambda}_{, A}=\boldsymbol{\pi}(\boldsymbol{\Lambda})
$$

We observe that the coefficient matrices are Hessian matrices and therefore symmetric.

By the definition of symmetric hyperbolicity due to Friedrichs [17] the system is symmetric hyperbolic, if there exists at least one co-vector $\zeta_{A}$ for which

$$
\frac{\partial^{2} h^{\prime A}}{\partial \boldsymbol{\Lambda} \partial \boldsymbol{\Lambda}} \zeta_{A} \sim \text { negative definite }\left(g^{A B} \zeta_{A} \zeta_{B}=1, \quad \zeta_{0}>0\right)
$$

In our case - with the concavity (5) of the entropy density $h_{\bar{\zeta}}$ for $\bar{\zeta}^{A}=-\frac{h^{\prime A}}{\sqrt{h^{\prime A} h_{A}^{\prime}}}-$ it is clear that such a co-vector exists. It is $\bar{\zeta}^{A}$ itself! Indeed we have

$$
\frac{\partial^{2}{h^{\prime}}^{A}}{\partial \boldsymbol{\Lambda} \partial \boldsymbol{\Lambda}} \bar{\zeta}_{A}=\frac{\partial^{2} h_{\bar{\zeta}}^{\prime}}{\partial \boldsymbol{\Lambda} \partial \boldsymbol{\Lambda}}+\frac{\partial \bar{\zeta}_{A}}{\partial \boldsymbol{\Lambda}} \frac{\partial{h^{\prime}}^{A}}{\partial \boldsymbol{\Lambda}} \sim \text { negative definite }
$$

by (62) and (58). Thus symmetric hyperbolicity is implied by the concavity of the entropy density both in the relativistic and the non-relativistic case.

It is true that in the relativistic case we have to rely on the privileged co-vector $\bar{\zeta}^{A}=-\frac{h^{\prime A}}{\sqrt{h^{\prime A} h_{A}^{\prime}}}$ in this context and therefore on a privileged Lorentz frame whose entropy density $h_{\bar{\zeta}}$ is concave in $\boldsymbol{F}_{\bar{\zeta}}$. The significance of this choice is not really understood. Indeed, we might have preferred the privileged frame to be the local rest frame of the body. In that respect it is reassuring that $h^{\prime^{A}}$ is often co-linear to the four-velocity $U^{A}$ as we shall see in Section 4.3 below; but not always! A better understanding is needed.

Note that in the non-relativistic case the only time-like co-vector is $\zeta_{A}=(1,0,0,0)$, a constant vector. In that case all the above-mentioned complications are absent: Concavity of the one and only entropy density $h^{0}$ is equivalent to symmetric hyperbolicity, see Section 3 above.

Also note that the requirement (65) of symmetric hyperbolicity ensures finite characteristic speeds, not necessarily speeds smaller than $c$ as we might have wished. [In this respect we may be tempted to replace Friedrich's definition of symmetric hyperbolicity by one of our own making, which might require (65) to be true for all time-like co-vectors $\boldsymbol{\zeta}_{A}$-instead of at least one. If we 
did that, we should anticipate the whole problem of speeds greater than $c$. Indeed, we recall the characteristic equation (15) which - for our system (64) - reads

$$
\operatorname{det}\left(\phi, A \frac{\partial^{2} h^{\prime A}}{\partial \boldsymbol{\Lambda} \partial \boldsymbol{\Lambda}}\right)=0 .
$$

If (65) were to hold for all time-like co-vectors $\zeta_{A}$, we could now conclude that $\phi_{, A}$ is space-like, or light-like, so that $g^{A B} \phi_{, A} \phi_{, B} \leq 0$ holds. Thus (12) would imply $V^{2} \leq c^{2}$. This is a clear case of assuming the desired result in a disguise and we do not follow this path.]

\subsection{Moments as four-fluxes and the vector potential}

Just like in the non-relativistic case the most plausible - and popular - choice of the four-fluxes $\boldsymbol{F}^{A}$ in relativistic thermodynamics is moments of the phase density $f(\boldsymbol{x}, \boldsymbol{p}, t)$ of the atoms, viz.

$$
F_{\alpha}^{A}=\int p^{A} p_{\alpha} f d \boldsymbol{P}, \quad(\alpha=1,2, \ldots n), \quad(A=0,1,2,3)
$$

This is formally identical to the non-relativistic case that was treated in Section $3^{4}$. There are essential differences, however

- $p^{A}$ is now the Lorentz vector of atomic four-momentum with $p^{0}>0$ and $p^{A} p_{A}=m^{2} c^{2}$. Accordingly $p_{\alpha}$ now stands for polynomials in the components of four-momentum. Thus instead of (27) we have

$$
p_{\alpha}=\left\{\begin{array}{l}
1 \\
p^{B_{1}} \\
p^{B_{1}} p^{B_{2}} \\
\vdots \\
p^{B_{1}} p^{B_{2}} \ldots p^{B_{N}}
\end{array}\right.
$$

- The element $d \boldsymbol{P}$ of phase space is now equal to $d \boldsymbol{p} / p_{0}$ instead of $d \boldsymbol{p}$.

Both are important differences. But many results from the non-relativistic theory will remain formally valid.

Thus for instance in the relativistic case we still have

$$
h^{\prime A}=\int p^{A} F(\chi) d P
$$

with

$$
F(\chi)=\mp k y \ln \left(1 \pm \mathrm{e}^{-\frac{\chi}{k}}\right)
$$

just like (33) and (39). We conclude that the vector potential $h^{\prime A}$ is not generally in the class of moments. However, in the non-degenerate limit, where $\mathrm{e}^{-\chi / k} \ll 1$ holds, we obtain from (69) (see also (41))

$$
F(\chi)=-k y \mathrm{e}^{-\frac{\chi}{k}} \quad \text { or } \quad F=-k f .
$$

Therefore $h^{\prime A}$ for a non-degenerate gas reads

$$
h^{\prime A}=-k \int p^{A} f d \boldsymbol{P}
$$

\footnotetext{
${ }^{4}$ The subtle differences between the non-relativistic moments (26) and the relativistic moments (67) may be studied in papers on the relativistic kinetic theory, e.g. Lichnerowicz \& Marrot [29], Chernikov [10, 11, 12] and Marle [32] also in the book by de Groot, van Leeuven \& van Weert [13].
} 
and that is in the class of moments. In fact $h^{\prime A}$ is equal to the four-velocity $\boldsymbol{U}^{A}$ of the gas to within a factor. We have

$$
h^{\prime A}=-\frac{n k}{c} U^{A},
$$

where $n$ is the number density of atoms in the rest frame of the gas.

We recall the discussion - in Section 4.2 - of the important role played by $h^{\prime A}$ in ensuring symmetric hyperbolicity of the field equations: Symmetric hyperbolicity was due to the concavity of $h_{\bar{\zeta}}\left(\boldsymbol{F}_{\bar{\zeta}}\right)$ in the privileged frame moving with the four-velocity $c \bar{\zeta}^{A}=-c \frac{h^{\prime A}}{\sqrt{h_{A}^{\prime} h^{\prime A}}}$. Now we see from (72) that - for the non-degenerate gas - we have $c \bar{\zeta}^{A}=U^{A}$ so that the privileged frame is the local rest frame of the gas. This is quite satisfactory, since the rest frame is naturally privileged. [There remains the question of why the rest frame is not the privileged one for a degenerate gas. This point is open and invites investigation.]

\subsection{Upper and lower bounds for the pulse speed}

We recall the form of the field equations (34)

$$
\left[\int p^{A} p_{\alpha} p_{\beta} \frac{d^{2} F}{d \chi^{2}} d \boldsymbol{P}\right] \Lambda_{\beta, A}=\pi_{\alpha}
$$

which is still valid in the relativistic case, albeit with $p^{A}$ as the Lorentz vector of the atomic fourmomentum rather than $p^{A}=\left(m c, p^{a}\right)$ as in Section 3. We already know that $\frac{d^{2} F}{d \chi^{2}}<0$ holds. Also $p^{A}$ is a time-like vector so that we have

$$
\zeta^{A} \int p^{A} p_{\alpha} p_{\beta} \frac{d^{2} F}{d \chi^{2}} d \boldsymbol{P} \sim \text { negative definite }
$$

for all time-like co-vectors $\zeta_{A}$.

Therefore the characteristic equation of the system (73) of field equations, viz.

$$
\operatorname{det}\left(\phi_{, A} \int \boldsymbol{p}^{A} p_{\alpha} p_{\beta} \frac{d^{2} F}{d \chi^{2}} d \boldsymbol{P}\right)=0
$$

implies that $\phi_{, A}$ is space-like, or light-like and therefore - by (12) - all characteristic speeds are smaller than $c$. We conclude that the speed of light is an upper bound for the pulse speed $V_{\max }$.

[Recall that the requirement (65) of symmetric hyperbolicity did not require speeds $\leq c$. I have discussed that point at the end of Section 4.2. Now, however, in extended thermodynamics of moments, because of the specific form of the vector potential, the condition (65) is satisfied for all co-vectors. Therefore all speeds are $\leq c$.]

More explicitly, by (11), the characteristic equation (75) reads

$$
\operatorname{det}\left(\int\left(p^{a} n_{a}-\frac{V}{c} p^{0}\right) p_{\alpha} p_{\beta} \frac{d^{2} F}{d \chi^{2}} d \boldsymbol{P}\right)=0
$$

and this holds in particular for $V_{\max }$. Obviously $\int p^{A} p_{\alpha} p_{\beta} \frac{d^{2} F}{d \chi^{2}} d \boldsymbol{P}$ is symmetric and $-\int p^{0} p_{\alpha} p_{\beta} \frac{d^{2} F}{d \chi^{2}} d \boldsymbol{P}$ is positive definite and symmetric. Therefore it follows from linear algebra (see Footnote (3)) that 


$$
\int\left(p^{a} n_{a}-\frac{V_{\max }}{c} p^{0}\right) p_{\alpha} p_{\beta} \frac{d^{2} F}{d \chi^{2}} d \boldsymbol{P} \sim \text { negative semi-definite. }
$$

In very recent papers, Boillat \& Ruggeri $[6,3]$ have used this knowledge to prove lower bounds for $V_{\max }$. The lower bounds depend on $n$, the number of fields, and for the number of fields tending to infinity the lower bound of $V_{\max }$ tends to $c$ from below. The strategy of proof is similar to the one employed in Section 3.6 for the non-relativistic case.

Therefore the pulse speeds of all moment theories are smaller than $c$, but they tend to $c$ as the number of moments tends to infinity. This result compares well with the corresponding result in Section 3.6 concerning the non-relativistic theory. In that case there was no upper bound so that the pulse speeds tended to infinity for extended thermodynamics of very many moments. 


\section{Relativistic Thermodynamics of Gases. 14-Field Theory}

While the synthetic treatment of the foregoing sections is concise and seems quite elegant, it is also little suggestive of the laws for heat flux and stress that we associate with non-equilibrium thermodynamics. Moreover, the elegance of this treatment disguises the fact that much work is needed in order to obtain specific results.

The following section highlights this situation by considering a viscous heat-conducting gas, a material which is fully characterized by 14 fields, viz. the density and flux of mass, energy and momentum, and stress and heat flux. With this choice of fields we shall be able to exploit the principle of relativity and the entropy inequality in explicit form and to calculate some specific pulse speeds.

It is true that much of the rigorous formal structure of the preceding section is lost when it comes to specific calculations. Linearization around equilibrium cannot be avoided, if we wish to obtain specific results, and that destroys global invertibility and general symmetric hyperbolicity. These properties are now restricted to situations close to equilibrium.

\subsection{Thermodynamic processes in viscous, heat-conducting gases}

The objective of thermodynamics of viscous, heat-conducting gases is the determination of the 14 fields

$$
\begin{aligned}
A^{A}: \text { particle flux vector } \\
A^{A B}: \text { energy-momentum tensor }
\end{aligned}
$$

in all events $x^{D}$. Both $A^{A}$ and $A^{A B}$ are Lorentz tensors. The energy-momentum tensor is assumed symmetric so that it has 10 independent components.

For the determination of these fields we need field equations and these are formed by the conservation laws of particle number and energy-momentum, viz.

$$
\begin{gathered}
A^{A}{ }_{, A}=0 \\
A^{A B}{ }_{, B}=0
\end{gathered}
$$

and by the equations of balance of fluxes

$$
A^{A B C}{ }_{C}=I^{A B} .
$$

$A^{A B C}$ is the flux tensor - it is completely symmetric -, and $I^{A B}$ is its production density. We assume

$$
I_{A}^{A}=0 \quad \text { and } \quad A_{B}^{A B}=c^{2} A^{A}
$$

so that among the 15 equations $(79,80,81)$ there are 14 independent ones, which is the appropriate number for 14 fields.

The components of $A^{A}$ and $A^{A B}$ have the following interpretations

$$
\begin{aligned}
& A^{0}: c \cdot \text { rest mass density, } \\
& A^{a}: \text { flux of rest mass, } \\
& A^{00}: \text { energy density, } \\
& A^{0 a}: 1 / c \cdot \text { energy flux, } \\
& A^{a 0}: c \cdot \text { momentum density, } \\
& A^{a b}: \text { momentum flux. }
\end{aligned}
$$

The motivation for the choice of equations $(79,80,81)$, and in particular $(81)$, stems from the kinetic theory of gases. Indeed $A^{A}$ and $A^{A B}$ are the first two moments in the kinetic theory and 
$A^{A}{ }_{, A}=0$ and $A^{A B}{ }_{, B}=0$ are the first two equations of transfer. Therefore it seems reasonable to take further equations from the equation of transfer for the third moment $A^{A B C}$ and these have the form (81). In the kinetic theory the two conditions (82) are satisfied.

The set of equations $(79,80,81)$ must be supplemented by constitutive equations for the flux tensor $A^{A B C}$ and the flux production $I^{A B}$. The generic form of these relations in a viscous, heat-conducting gas reads

$$
\begin{aligned}
A^{A B C} & =\hat{A}^{A B C}\left(A^{M}, A^{M N}\right) \\
I^{A B} & =\hat{I}^{A B}\left(A^{M}, A^{M N}\right) .
\end{aligned}
$$

If the constitutive functions $\hat{A}$ and $\hat{I}$ are known, we may eliminate $A^{A B C}$ and $I^{B C}$ between (79, 80,81 ) and (84) and obtain a set of field equations for $A^{M}, A^{M N}$. Each solution is called a thermodynamic process.

It is clear upon reflection that this theory, based on $(79,80,81)$ and $(84)$, provides a special case of the generic structure explained in Section 2.

\subsection{Constitutive theory}

We recall the restrictive principles of the constitutive theory from Section 2 and adjust them to the present case

- entropy inequality $h^{A}{ }_{, A} \geq 0$ with $h^{A}=\hat{h}^{A}\left(A^{M}, A^{M N}\right)$,

- principle of relativity.

The former principle was discussed and exploited in the general scheme of Section 2, but the principle of relativity was not. This principle assumes that the constitutive functions $\hat{A}^{A B C}, \hat{I}^{A B}$, $\hat{h}^{A}$ - generically $\hat{C}$ - are invariant under Lorentz transformations

$$
\stackrel{*}{x}^{A}=\stackrel{*}{x}^{A}\left(x^{B}\right) \text {. }
$$

Thus the principle of relativity may be stated in the form

$$
C=\hat{C}\left(A^{M}, A^{M N}\right) \quad \text { and } \quad \stackrel{*}{C}=\hat{C}\left(\stackrel{*}{A}{ }^{M}, \stackrel{*}{A}{ }^{M M}\right)
$$

Note that $\hat{C}$ is the same function in both equations.

It is complicated and cumbersome to exploit the constitutive theory but the results are remarkably specific, at least for near-equilibrium processes:

- $\hat{A}^{A B C}$ will be reduced to the thermal equation of state.

- $\hat{I}^{A B}$ will be reduced to the relaxation times of the gas, which may be considered to be of the order of magnitude of the mean time of free flight of its molecules.

For details of the calculation the reader is referred to the literature, in particular to the book by Müller \& Ruggeri [39, 40] or the paper by Liu, Müller \& Ruggeri [31]. Here we explain only the results.

\subsection{Results of the constitutive theory}

No matter how much a person may be conditioned to think relativistically, he will appreciate the decomposition of the four-tensors $A^{A}, A^{A B}$ and $h^{A}$ into their suggestive time-like and space-like 
components. We have

$$
\begin{aligned}
A^{A} & =n m U^{A} \\
A^{A B} & =t^{\langle A B\rangle}+(p(n, e)+\pi) h^{A B}+\frac{1}{c^{2}}\left(U^{A} q^{B}+U^{B} q^{A}\right)+\frac{e}{c^{2}} U^{A} U^{B} \\
h^{A} & =h U^{A}+\Phi^{A},
\end{aligned}
$$

and the components have suggestive meaning as follows

$$
\begin{aligned}
n & : \text { number density } \\
U^{A} & : \text { velocity } \\
t^{\langle A B\rangle} & : \text { stress deviator } \\
p+\pi & : \text { pressure } \\
q^{A} & : \text { heat flux } \\
e & : \text { energy density } \\
h & : \text { entropy density } \\
\Phi^{A} & : \text { (non-convective) entropy flux. }
\end{aligned}
$$

At least this is how $n$ through $\Phi^{A}$ are to be interpreted in the rest frame of the gas.

We have defined $h^{A B}=\frac{1}{c^{2}} U^{A} U^{B}-g^{A B}$ and $m$ is the molecular rest mass.

The decomposition (86) is not only popular because of its intuitive quality but also, since it is now possible to characterize equilibrium as a process in which the stress deviator $t^{\langle A B\rangle}$, the heat flux $q^{A}$ and the dynamic pressure $\pi$ - the non-equilibrium part of the pressure - vanish.

The equilibrium pressure $p$ is a function of $n$ and $e$, the thermal equation of state. In thermodynamics it is often useful to replace the variables $(n, e)$ by

$$
\text { fugacity } \alpha \text { and absolute temperature } T \text {, }
$$

because these two variables can be measured - at least in principle. Also $\alpha$ and $T$ are the natural variables of statistical thermodynamics which provides the thermal equation of state in the form $p=p(\alpha, T)$. The transition between the new variables $(\alpha, T)$ and the old ones $(n, e)$ can be effected by the relations

$$
n m=-\frac{1}{T} \dot{p} \quad \text { and } \quad e=p^{\prime}-p
$$

where 'and ' here and below denote differentiation with respect to $\alpha$ and $\ln T$ respectively.

If we restrict attention to a linear theory in $t^{\langle A B\rangle}, q^{A}$, and $\pi$, we can satisfy the principle of relativity with linear isotropic functions for $A^{A B C}, I^{B C}$ viz.

$$
\begin{aligned}
A^{A B C}= & \left(C_{1}^{0}+C_{1}^{\pi} \pi\right) U^{A} U^{B} U^{C}+\frac{c^{2}}{6}\left(n m-C_{1}^{0}-C_{1}^{\pi} \pi\right) . \\
& \cdot\left(g^{A B} U^{C}+g^{B C} U^{A}+g^{C A} U^{B}\right)+C_{3}\left(g^{A B} q^{C}+g^{B C} q^{A}+g^{C A} q^{B}\right)- \\
& -\frac{6}{c^{2}} C_{3}\left(U^{A} U^{B} q^{C}+U^{B} U^{C} q^{A}+U^{C} U^{A} q^{B}\right)+ \\
& +C_{5}\left(t^{\langle A B\rangle} U^{C}+t^{\langle B C\rangle} U^{A}+t^{\langle C A\rangle} U^{B}\right), \\
I^{B C}= & B_{1}^{\pi} \pi g^{A B}-\frac{4}{c^{2}} B_{1}^{\pi} \pi U^{A} U^{B}+B_{3} t^{\langle A B\rangle}+\frac{1}{c^{2}} \hat{B}^{4}\left(q^{A} U^{B}+q^{B} U^{A}\right) .
\end{aligned}
$$

Note that $I^{B C}$ vanishes in equilibrium so that no entropy production occurs in that state. The coefficients $C$ and $B$ in $(89,90)$ are functions of $e$ and $n$, or $\alpha$ and $T$. In fact, the entropy principle 
determines the $C$ 's fully in terms of the thermal equation of state $p=p(\alpha, T)$ as follows

$$
\begin{aligned}
& C_{1}^{0}=\frac{\Gamma_{1}^{\prime}}{2 c^{2} T} \quad \text { with } \Gamma_{1}=-2 c^{2} T^{6} \int \frac{\dot{p}}{T^{7}} d T \\
& C_{1}^{\pi}=-\frac{2}{c^{2} T} \frac{\left[\begin{array}{ccc}
-\ddot{p} & \dot{p}-\dot{p}^{\prime} & \dot{\Gamma}_{1} \\
\dot{p}-\dot{p}^{\prime} & p^{\prime}-p^{\prime \prime} & \Gamma_{1}^{\prime}-\Gamma_{1} \\
\dot{\Gamma}_{1} & \Gamma_{1}^{\prime}-\Gamma_{1} & \frac{5}{3} \Gamma_{2}
\end{array}\right]}{\left[\begin{array}{ccc}
-\ddot{p} & \dot{p}-\dot{p}^{\prime} & \dot{\Gamma}_{1} \\
\dot{p}-\dot{p}^{\prime} & p^{\prime}-p^{\prime \prime} & \Gamma_{1}^{\prime}-\Gamma_{1} \\
-\dot{p} & -p^{\prime} & \frac{5}{3} \Gamma_{1}
\end{array}\right]} \\
& C_{3}=-\frac{1}{2 T} \frac{\left[\begin{array}{cc}
\dot{p} & -\dot{\Gamma}_{1} \\
\Gamma_{1} & \Gamma_{2}
\end{array}\right]}{\left[\begin{array}{cc}
\dot{p} & -\dot{\Gamma}_{1} \\
p^{\prime} & \Gamma_{1}-\Gamma_{1}^{\prime}
\end{array}\right]} \\
& C_{5}=-\frac{1}{2 T} \frac{\Gamma_{2}}{\Gamma_{1}} \quad \text { with } \Gamma_{2}=2 c^{2} T^{8} \int \frac{1}{T^{3}} \dot{\Gamma}_{1} d T \text {. }
\end{aligned}
$$

The $B$ 's in (90) are restricted by inequalities, viz.

$$
B_{1}^{\pi} \geq 0, \quad \hat{B}_{4} \geq 0, \quad B_{3} \leq 0
$$

All $B$ 's have the dimension 1 /sec and we may consider them to be of the order of magnitude of the collision frequency of the gas molecules.

In conclusion we may write the field equations in the form

$$
\begin{gathered}
\left(n m U^{A}\right)_{, A}=0 \\
\left(t^{\langle B A\rangle}+(p+\pi) h^{B A}+\frac{1}{c^{2}}\left(q^{B} U^{A}+q^{A} U^{B}\right)+\frac{e}{c^{2}} U^{B} U^{A}\right)_{, A}=0 \\
A^{B C A}{ }_{, A}=B_{1}^{\pi} \pi g^{B C}-\frac{4}{c^{2}} B_{1}^{\pi} \pi U^{B} U^{C}+B_{3} t^{\langle B C\rangle}+\frac{1}{c^{2}} \hat{B}_{4}\left(q^{B} U^{C}+q^{C} U^{B}\right),
\end{gathered}
$$

where $A^{B C A}$ must be inserted from (89) and (91). This set of equations represents the field equations of extended thermodynamics. We conclude that extended thermodynamics of viscous, heat-conducting gases is quite explicit - provided we are given the thermal equation of state $p=p(\alpha, T)$ - except for the coefficients $B$. These coefficients must be measured and we proceed to show how.

\subsection{The laws of Navier-Stokes and Fourier}

It is instructive to identify the classical constitutive relations of Navier-Stokes and Fourier of TIP within the scheme of extended thermodynamics. They are obtained from $(93,94,95)$ by the first step of the so-called Maxwell iteration which proceeds as follows: The $n^{\text {th }}$ iterate

$$
\stackrel{(n)}{I} A B(\text { or } \stackrel{(n)}{\pi}, \stackrel{(n)}{t} A B \stackrel{(n)}{q} A)
$$

results from 


$$
\begin{aligned}
& A^{A}{ }_{, A}=0 \\
& { }^{(n-1)}{ }_{A B}{ }_{, B}=0 \\
& \stackrel{(n-1)}{A} A B C{ }_{, C}={ }_{I}^{(n)} A B
\end{aligned}
$$

with the initiation agreement

$$
\begin{aligned}
& \stackrel{(E)}{A}^{A}{ }_{A}=0 \\
& { }_{A}^{(E)}{ }_{A B}{ }_{B}=0 \\
& \stackrel{(E)}{A}^{A B C}{ }_{, C}=\stackrel{(1)}{I}^{A B}
\end{aligned}
$$

where $\stackrel{(E)}{A}$ are equilibrium values.

A little calculation provides the first iterates for dynamic pressure, stress deviator and heat flux in the form

$$
\begin{gathered}
\stackrel{(1)}{\pi}=-\lambda\left[U^{A}, A\right] \\
\stackrel{(1)}{t}\langle A B\rangle=\mu\left[h_{A}^{M} h_{B}^{N} U_{\langle M, N\rangle}\right] \\
q_{A}=-\kappa\left[h_{A}^{M}(\ln T), M-\frac{1}{c^{2}} \frac{d U_{M}}{d \tau}\right]
\end{gathered}
$$

with

$$
\begin{aligned}
& \lambda=\frac{1}{2 T B_{1}^{\pi}} \frac{\left[\begin{array}{ccc}
-\ddot{p} & \dot{p}-\dot{p}^{\prime} & \dot{\Gamma}_{1} \\
\dot{p}-\dot{p}^{\prime} & p^{\prime}-p^{\prime \prime} & \Gamma_{1}^{\prime}-\Gamma_{1} \\
\dot{\Gamma}_{1} & \Gamma_{1}^{\prime}-\Gamma_{1} & \frac{5}{3} \Gamma_{2}
\end{array}\right]}{\left[\begin{array}{cc}
-\ddot{p} & \dot{p}-\dot{p}^{\prime} \\
\dot{p}-\dot{p}^{\prime} & p^{\prime}-p^{\prime \prime}
\end{array}\right]} \\
& \mu=\frac{1}{2 T B_{3}} \Gamma_{1} \\
& \kappa=\frac{1}{2 T^{2} \hat{B}_{4}} \frac{\left[\begin{array}{ll}
\dot{p} & -\dot{\Gamma}_{1} \\
p^{\prime} \Gamma_{1}-\Gamma_{1}^{\prime}
\end{array}\right]}{\dot{p}}
\end{aligned}
$$

These are the relativistic analogues of the classical phenomenological equations of Navier-Stokes and Fourier. $\lambda, \mu$ and $\kappa$ are the bulk viscosity, the shear viscosity and the thermal conductivity respectively; all three of these transport coefficients are non-negative by the entropy inequality.

The only essential difference between the equations $(97,98,99)$ and the non-relativistic phenomenological equations is the acceleration term in (99). This contribution to the Fourier law was first derived by Eckart, the founder of thermodynamics of irreversible processes. It implies that the temperature is not generally homogeneous in equilibrium. Thus for instance equilibrium of a gas in a gravitational field implies a temperature gradient, a result that antedates even Eckart.

We have emphasized that the field equations of extended thermodynamics should provide finite speeds. Below in Section 5.6 we shall give the values of the speeds for non-degenerate gases. In contrast TIP leads to parabolic equations whose fastest characteristic speeds are always infinite. Indeed, if the phenomenological equations $(97,98,99)$ are introduced into the conservation laws (93, 94) of particle number, energy and momentum, we obtain a closed system of parabolic equations for $n, U_{A}$ and $e$. This unwelcome feature results from the Maxwell iteration; it persists to arbitrarily high iterates.

\subsection{Specific results for a non-degenerate relativistic gas}

For a relativistic gas Jüttner $[22,23]$ has derived the phase density for Bosons and Fermions, namely

$$
f_{E}=\frac{y}{\exp \left[\frac{m}{k} \alpha+\frac{U_{A}}{k T} p^{A}\right] \mp 1} \quad \text { or } \quad f_{E}=\frac{y}{\exp \left[\frac{m}{k} \alpha+\frac{m c^{2}}{k T} \sqrt{1+\frac{p^{2}}{m^{2} c^{2}}}\right] \mp 1}
$$


The latter equation is valid in the rest frame of the gas. $p^{A}$ is the atomic four-momentum and we have $p_{A} p^{A}=m^{2} c^{2}$. Jüttner has used these phase densities to calculate the equations of state. For the non-degenerate gas he found that Bessel functions of the second kind, viz.

$$
K_{n}\left(\frac{m c^{2}}{k T}\right)=\int_{0}^{\infty} \cos h(n \rho) \exp \left(-\frac{m c^{2}}{k T} \cos h \rho\right) d \rho
$$

are the relevant special functions. The thermal equation of state $p=p(\alpha, T)$ reads

$$
p=n k T \quad \text { with } \quad n=\exp \left(-\frac{m}{k} \alpha\right) \cdot 4 \pi y m^{3} c^{3} \frac{K_{2}\left(\frac{m c^{2}}{k T}\right)}{\frac{m c^{2}}{k T}}
$$

where $1 / y$ is the smallest phase space element. From (102) we obtain with $G=\frac{K_{3}}{K_{2}}$ and $\gamma=\frac{m c^{2}}{k T}$

$$
e=n m c^{2}\left(G-\frac{1}{\gamma}\right), \quad \frac{\Gamma_{1}}{T}=n m c^{2} \frac{2}{\gamma} G
$$

and hence

$$
\begin{aligned}
C_{1}^{0} & =n m\left(1+\frac{6}{\gamma} G\right) \\
C_{1}^{\pi} & =-\frac{6}{c^{2}} \frac{\left(2-\frac{5}{\gamma^{2}}\right)+\left(\frac{19}{\gamma}-\frac{30}{\gamma^{3}}\right) G-\left(2-\frac{45}{\gamma^{2}}\right) G^{2}-\frac{9}{\gamma} G^{3}}{\frac{3}{\gamma}-\left(2-\frac{20}{\gamma^{2}}\right) G-\frac{13}{\gamma} G^{2}+2 G^{3}} \\
C_{3} & =-\frac{1}{\gamma} \frac{1+\frac{6}{\gamma} G-G^{2}}{1+\frac{5}{\gamma} G-G^{2}} \\
C_{5} & =\left(\frac{6}{\gamma}+\frac{1}{G}\right) .
\end{aligned}
$$

The transport coefficients read

$$
\begin{aligned}
\lambda & =\frac{1}{3} \frac{n m c^{2}}{\gamma} \frac{1}{B_{1}^{\pi}} \frac{-\frac{3}{\gamma}+\left(2-\frac{20}{\gamma^{2}}\right) G+\frac{13}{\gamma} G^{2}+2 G^{3}}{1-\frac{1}{\gamma^{2}}+\frac{5}{\gamma} G-G^{2}} \\
\mu & =-n k T \frac{1}{B_{3}} G \\
\kappa & =-n k T \frac{c^{2}}{\hat{B}_{4}}\left(\gamma+5 G-\gamma G^{2}\right) .
\end{aligned}
$$

It is instructive to calculate the leading terms of the transport coefficients in the non-relativistic case $m c^{2} \gg k T$. We obtain

$$
\begin{gathered}
\lambda=-\frac{5}{6 B_{1}^{\pi}} n k T \frac{1}{\gamma^{2}} \\
\mu=-\frac{1}{B_{3}} n k T \\
\kappa=-\frac{5}{2 \hat{B}_{4}} \frac{n k^{2} T}{m} .
\end{gathered}
$$

It follows that the bulk viscosity does not appear in a non-relativistic gas. Recall that the coefficients $1 / B$ are relaxation times of the order of magnitude of the mean-time of free flight; so they are not in any way "relativistically small".

Note that $\lambda, \mu$ and $\kappa$ are measurable, at least in principle, so that the $B$ 's may be calculated from (105). Therefore it follows that the constitutive theory has led to specific results. All constitutive coefficients are now explicit: The $C$ 's can be calculated from the thermal equation of state $p=p(\alpha, T)$ and the $B$ 's may be measured. 
It might seem from (106) and (97) that the dynamic pressure is of order $O\left(\frac{1}{\gamma^{2}}\right)$ but this is not so as was recently discovered by Kremer \& Müller [27]. Indeed, the second step in the Maxwell iteration for $\pi$ provides a term that is of order $O\left(\frac{1}{\gamma}\right)$, see also [28]. That term is proportional to the second gradient of the temperature $T$ so that it may be said to be due to heating or cooling.

Specific results of the type $(104,105)$ can also be calculated for degenerate gases with the thermal equation of state $p(\alpha, T)$ for such gases. That equation was also derived by Jüttner [23]. The results for 14 fields may be found in Müller \& Ruggeri [39, 40].

\subsection{Characteristic speeds in a viscous, heat-conducting gas}

We recall from Section 2.4, in particular (14), that the jumps $\delta \boldsymbol{u}$ across acceleration waves and their speeds of propagation are to be calculated from the homogeneous system

$$
\phi_{, A} \frac{\partial \boldsymbol{F}^{A}}{\partial \boldsymbol{u}} \delta \boldsymbol{u}=0 .
$$

In the present context, where the field equations are given by $(79,80)$ this homogeneous algebraic system spreads out into three equations, viz.

$$
\phi,{ }_{A} \delta A^{A}=0, \quad \phi_{, A} \delta A^{A B}=0, \quad \phi_{, A} \delta A^{A B C}=0 .
$$

By (89) and (91) this is a fully explicit system, if the thermal equation of state $p=p(\alpha, T)$ is known. The vanishing of its determinant determines the characteristic speeds. Seccia \& Strumia [44] have calculated these speeds - one transversal and two longitudinal ones - for non-degenerate gases and obtained the following results in the non-relativistic and ultra-relativistic cases

$$
\begin{array}{lll}
\frac{m c^{2}}{k T} \gg 1: V_{\text {trans }}=\sqrt{\frac{7 k}{5 m} T}, & V_{\text {long }}^{1}=\sqrt{\frac{4 k}{3 m} T}, & V_{\text {long }}^{2}=\sqrt{5.18 \frac{k}{m} T}, \\
\frac{m c^{2}}{k T} \ll 1: V_{\text {trans }}=\sqrt{\frac{1}{5}} c, & V_{\text {long }}^{1}=\sqrt{\frac{1}{3}} c, & V_{\text {long }}^{2}=\sqrt{\frac{3}{5}} c .
\end{array}
$$

All speeds are finite and smaller than $c$. Inspection shows that in the non-relativistic limit the order of magnitude of these speeds is that of the ordinary speed of sound, while in the ultra-relativistic case the speeds come close to $c$.

\subsection{Discussion}

So as to anticipate a possible misunderstanding I remark that the equations $(93,94,95)$ with $A^{A B A}$ from (89) and (91) are neither symmetric nor fully hyperbolic. Indeed the underlying symmetry of the system $(79,80,81)$, and (84) reveals itself only when the Lagrange multipliers $\boldsymbol{\Lambda}$ are used as variables. But $(93,94,95,89,91)$ are equations for the physical variables $A^{A}, A^{A B}$ or in fact $n, T, U^{A}, t^{A B}$, and $q^{A}$. Also the hyperbolicity in the whole state space is lost, because the equations $(89,90)$ are restricted to linear terms. Therefore the system is hyperbolic only in the neighbourhood of equilibrium. For a more detailed discussion of these aspects, see Müller \& Ruggeri [39, 40]. 


\section{References}

[1] Boillat, G., "Sur l'existence et la recherche d'équations de conservation supplementaires pour les hyperbolique.", C. R. Acad. Sci. Ser. A, 278, (1974). 3.2

[2] Boillat, G., "Wave velocities in relativistic extended thermodynamics", in Müller, I., and Ruggeri, T., eds., Kinetic Theory and Extended Thermodynamics, Proceedings of the ISIMM Symposium, Bologna, May 18-20, 1987, (Pitagora Editrice, Bologna, 1987). 1

[3] Boillat, G., and Ruggeri, T., "Maximum Wave Velocity of Degenerate and Non-Degenerate Relativistic Gases", in preparation. 1, 4.4

[4] Boillat, G., and Ruggeri, T., "Hyperbolic Principal Subsystems: Entropy Convexity and Subcharacteristic Conditions", Arch. Ration. Mech. Anal., 137, 305-320, (1997). [DOI]. 1

[5] Boillat, G., and Ruggeri, T., "Moment equations in the kinetic theory of gases and wave velocities", Continuum Mech. Thermodyn., 9, 205-212, (1997). [DOI]. 1, 3.6

[6] Boillat, G., and Ruggeri, T., "Maximum wave velocity in the moments system of a relativistic gas", Continuum Mech. Thermodyn., 11, 107-111, (1999). [DOI]. 1, 4.4

[7] Cattaneo, C., "Sulla conduzione del calore", Atti Semin. Mat. Fis. Univ. Modena, 3, (1948). 1

[8] Cercignani, C., "Speed of propagation of infinitesimal disturbances in a relativistic gas", Phys. Rev. Lett., 50, 1122-1124, (1983). [DOI]. 1

[9] Cercignani, C., and Majorana, A., "Analysis of thermal and shear waves according to BKG kinetic model", Z. Angew. Math. Phys., 36, 699-711, (1985). [DOI]. 1

[10] Chernikov, N.A., "The relativistic gas in the gravitational field", Acta Phys. Pol., 23, 629-645, (1963). 4

[11] Chernikov, N.A., "Equilibrium distribution of the relativistic gas", Acta Phys. Pol., 26, 10691092, (1964). 4

[12] Chernikov, N.A., "Microscopic foundation of relativistic hydrodynamics", Acta Phys. Pol., 27, 465-489, (1965). 4

[13] de Groot, S.R., van Leeuwen, W.A., and van Weert, C.G., Relativistic Kinetic Theory: Principles and Applications, (North-Holland; Elsevier, Amsterdam; New York, 1980). 4

[14] Eckart, C., "The Thermodynamics of Irreversible Processes. I. The Simple Fluid", Phys. Rev., 58, 267-269, (1940). [DOI]. 1

[15] Eckart, C., "The Thermodynamics of Irreversible Processes. II. Fluid Mixtures", Phys. Rev., 58, 269-275, (1940). [DOI]. 1

[16] Eckart, C., "The Thermodynamics of Irreversible Processes. III. Relativistic Theory of the Simple Fluid", Phys. Rev., 58, 919-924, (1940). [DOI]. 1

[17] Friedrichs, K.O., "On the laws of relativistic electro-magneto-fluid dynamics", Commun. Pure Appl. Math., 27, 749-808, (1974). 4.2

[18] Friedrichs, K.O., and Lax, P.D., "Systems of Conservation Equations with a Convex Extension", Proc. Natl. Acad. Sci. USA, 68, 1686-1688, (1971). [DOI]. 3.2 
[19] Godunov, S.K., "An interesting class of quasi-linear systems", Sov. Math. Dokl., 2, 947-949, (1961). 3.2

[20] Huang, K., Statistical Mechanics, (Wiley, New York, 1963). 3.4

[21] Israel, W., "Nonstationary irreversible thermodynamics: A causal relativistic theory", Ann. Phys. (N.Y.), 100, 310-331, (1976). [DOI]. 1

[22] Jüttner, F., "Das Maxwellsche Gesetz der Geschwindigkeitsverteilung in der Relativtheorie", Ann. Phys. (Leipzig), 339, 856-882, (1911). [DOI]. 5.5

[23] Jüttner, F., "Die relativistische Quantentheorie des idealen Gases", Z. Phys., 47, 542-566, (1928). [DOI]. 5.5, 5.5

[24] Kranys, M., "Phase and signal velocities of waves in dissipative media. Special relativistic theory", Arch. Ration. Mech. Anal., 48, 274-301, (1972). [DOI]. 1

[25] Kremer, G.M., Zur erweiterten Thermodynamik idealer und dichter Gase, Ph.D. Thesis, (Technische Universität, Berlin, 1985). 1

[26] Kremer, G.M., "Extended thermodynamics of ideal gases with 14 fields", Ann. Inst. Henri Poincare A, 45, 419-440, (1986). Related online version (cited on 25 May 2009):

http://www. numdam.org/item?id=AIHPA_1986_-45_4_419_0. 1

[27] Kremer, G.M., and Müller, I., "Dynamic pressure in relativistic thermodynamics", Ann. Inst. Henri Poincare A, 67, 111-121, (1997). Related online version (cited on 25 May 2009): http://www . numdam.org/item?id=AIHPA_1997_-67_2_111_0. 5.5

[28] Kremer, G.M., and Müller, I., "Linearized Burnett equation for the dynamic pressure of a relativistic gas", Continuum Mech. Thermodyn., 10, 49-53, (1998). [DOI]. 5.5

[29] Lichnerowicz, A., and Marrot, R., "Propriétés statistiques des ensembles de particules en relativité restreinte", C. R. Acad. Sci., 210, 759-761, (1940). 4

[30] Liu, I.-S., "Method of Lagrange multipliers for exploitation of the entropy principle", Arch. Ration. Mech. Anal., 46, 131-148, (1972). [DOI]. 2.3

[31] Liu, I.-S., Müller, I., and Ruggeri, T., "Relativistic thermodynamics of gases", Ann. Phys. (N.Y.), 169, 191-219, (1986). [DOI]. 1, 5.2

[32] Marle, C., "Sur l'établissement des équations de l'hydrodynamique des fluides relativistes dissipatifs. I. - L'équation de Boltzmann relativiste", Ann. Inst. Henri Poincare A, 10(1), 67-126, (1969). Related online version (cited on 25 May 2009):

http://www.numdam.org/item?id=AIHPA_1969_-10_1_67_0. Part II: 10(2), 127-194, 1969. 4

[33] Maxwell, J.C., "On the Dynamical Theory of Gases", Philos. Trans. R. Soc. London, 157, 49-88, (1867). [DOI]. 1

[34] Maxwell, J.C., "On Stresses in Rarified Gases Arising from Inequalities of Temperature", Philos. Trans. R. Soc. London, 170, 231-256, (1879). [DOI]. 1

[35] Müller, I., Zur Ausbreitungsgeschwindigkeit von Störungen in kontinuierlichen Medien, Ph.D. Thesis, (RWTH Aachen, Aachen, Germany, 1966). 1

[36] Müller, I., "On the entropy inequality", Arch. Ration. Mech. Anal., 26, 118-141, (1967). [DOI]. 2.2 
[37] Müller, I., "Zum Paradoxon der Wärmeleitungstheorie", Z. Phys., 198, 329-344, (1967). [DOI]. 1

[38] Müller, I., "Toward relativistic thermodynamics", Arch. Ration. Mech. Anal., 34, 259-282, (1969). [DOI]. 2.2

[39] Müller, I., and Ruggeri, T., Extended Thermodynamics, Springer Tracts in Natural Philosophy, vol. 37, (Springer, New York, 1993). 1, 5.2, 5.5, 5.7

[40] Müller, I., and Ruggeri, T., Rational Extended Thermodynamics, Springer Tracts in Natural Philosophy, vol. 37, (Springer, New York, 1998), 2nd edition. 1, 5.2, 5.5, 5.7

[41] Ruggeri, T., "Galilean invariance and entropy principle for systems of balance laws. The structure of extended thermodynamics", Continuum Mech. Thermodyn., 1, 3-20, (1989). [DOI]. 1

[42] Ruggeri, T., "Convexity and symmetrization in relativistic theories", Continuum Mech. Thermodyn., 2, 163-177, (1990). 4

[43] Ruggeri, T., and Strumia, A., "Main field and convex covariant density for quasi-linear hyperbolic systems: Relativistic fluid dynamics", Ann. Inst. Henri Poincare A, 34, 65-84, (1981). Related online version (cited on 25 May 2009): http://www.numdam.org/item?id=AIHPA_1981__34_1_65_0. 1, 3.2

[44] Seccia, L., and Strumia, A., "Wave propagation in relativistic extended thermodynamics", Continuum Mech. Thermodyn., 2, 151-161, (1990). [DOI]. 1, 5.6

[45] Sirovich, L., and Thurber, J.K., "Sound Propagation According to the Kinetic Theory of Gases", in Laurman, J.A., ed., Rarefied Gas Dynamics, Vol. 1, Proceedings of the 3rd International Symposium, Paris 1962, p. 159, (Academic Press, New York, 1963). 1

[46] Stewart, J.M., "On transient relativistic thermodynamics and kinetic theory", Proc. R. Soc. London, Ser. A, 357, 59-75, (1977). [DOI]. 1

[47] Struchtrup, H., "An Extended Moment Method in Radiative Transfer: The Matrices of Mean Absorption and Scattering Coefficients", Ann. Phys. (N.Y.), 257, 111-135, (1997). [DOI]. 1

[48] Wang Chang, C.S., and Uhlenbeck, G.E., "The Kinetic Theory of Gases", in De Boer, J., and G.E., Uhlenbeck, eds., The Kinetic Theory of Gases. The dispersion of sound in monoatomic gases, Studies in Statistical Mechanics, vol. 5, (North-Holland, Amsterdam, 1970). 1

[49] Weiss, W., Zur Hierarchie der Erweiterten Thermodynamik, Ph.D. Thesis, (Technische Universität, Berlin, 1990). 1, 3.5

[50] Weiss, W., "Continuous shock structure in extended thermodynamics", Phys. Rev. E, 52, R5760-R5763, (1995). [DOI]. 1

[51] Weiss, W., and Müller, I., "Light scattering and extended thermodynamics", Continuum Mech. Thermodyn., 7, 123-177, (1995). [DOI]. 1 